\title{
Effect of HF and Plasma Treated Glass Surface on Vapor Phase-Polymerized Poly(3,4-ethylenedioxythiophene) Thin Film : Part II
}

\author{
Joonwoo Lee and Sungsoo Kim ${ }^{\dagger}$
}

\begin{abstract}
In this study, in order to investigate how consecutive treatments of glass surface with HF acid and water vapor/Ar plasma affect the quality of 3-aminopropyltriethoxysilane self-assembled monolayer (APS-SAM), poly(3,4ethylenedioxythiophene) (PEDOT) thin films were vapor phase-polymerized immediately after spin coating of FeCl3 and poly-urethane diol-mixed oxidant solution on the monolayer surfaces prepared at various treatment conditions. For the film characterization, various poweful tools were used, e.g., FE-SEM, an optical microscope, four point probe, and a contact angle analyzer. The characterization revealed that a well prepared APS-SAM on a glass surface treated with water vapor/Ar plasma is very useful for uniform coating of $\mathrm{FeCl} 3$ and DUDO mixed oxidant solution, regardless of HF treatment. On the other hand, a bare glass surface without APS-SAM but treated with HF and water vapor/Ar plasma generally led to a very poor oxidant film. As a result, PEDOT films vapor phase-polymerized on APS-SAM surfaces are far superior to those on bare glass surfaces in the quality and electrical characteristics aspects.
\end{abstract}

Keywords: Poly(3,4-ethylenedioxythiophene), Vapor Phase Polymerization, 3-Aminopropyltriethoxysilane, Water Vapor/ Argon Plasma

\section{Introduction}

For decades, conductive p-conjugated polymers have fascinated many scientists with their great potential for applicability to the next-generation flexible display device such as organic thin film transistors (OTFTs) and organic light emitting diodes (OLEDs) ${ }^{[1]}$. Among these polymers, poly(3,4-ethylenedioxythiophene) (PEDOT) has been particularly focused on as a potential component of OTFT or OLED devices due to its superb material characteristics ${ }^{[2]}$. PEDOT is electrochemically very stable, and it has a low bandgap energy $(1.5 \sim 1.7 \mathrm{eV})$ and a considerably high electrical conductivity. Highly conductive PEDOT thin film is generally synthesized via two different but consecutive procedures, i.e., oxidant spin-coating and oxidative vapour phase polymerization (VPP). Electrical characteristics of the film prepared by the serial process are critically affected by its growth morphology. In particular, our recent study

Department of Nano-Polymer Materials Engineering, Pai Chai University, Daejeon 302-735, Korea

${ }^{\dagger}$ Corresponding author : skim@pcu.ac.kr

(Received : November 24, 2013, Revised : December 13, 2013, Accepted : December 20, 2013) showed that the morphology of PEDOT thin film polymerized with $\mathrm{FeCl}_{3}$ alone and grown on a high quality 3-amonopropyltriethoxysilane self-assembled monolayer (APS-SAM) is noticeably different from that of the thin film grown on a relatively low quality APS$\mathrm{SAM}^{[3]}$. The film grown on the high quality SAM by vapour phase polymerization (VPP) method is denser (or less porous) and more robustly adhered to oxidized silicon wafer surface. It suggests that APS-SAM helps to improve the PEDOT film quality, thereby improving the electrical characteristics of the film. Therefore, in the same context, it is fair to think that the quality of APS monolayer self-assembled on a bare glass surface is critical for smooth and uniform coating of the oxidant on the same surface. In general, silanol $(\mathrm{Si}-\mathrm{OH})$ density on a silicon oxide type surface is a crucial factor in highly dense and uniform self assembly of alkylsilane monolayer. Accordingly, in this study, to maximally introduce silanol groups to glass surface, the substrate surface was consecutively treated with hydrofluoric acid and water vapor/Ar plasma. Then, APS monolayer was self assembled on diverse surfaces treated under several different conditions. Quality of oxidant and PEDOT films on the monolayer surfaces was investigated and 
the effect of HF and plasma treatments on the film quality was also verified.

\section{Experimental Section}

Glass samples $(1.5 \mathrm{~cm} \times 1.5 \mathrm{~cm})$ were purchased from Hyunil Lab-Mate Corporation, Korea. 3-Aminopropyltriethoxysilane (APS), Iron (III) Chloride hexa-hydrate $\left(\mathrm{FeCl}_{3}-6 \mathrm{H}_{2} \mathrm{O}\right.$ ), Polyurethane diol (DUDO), and 3,4-Ethylenedioxythiophene (EDOT) were purchased from Sigma-Aldrich Inc., USA and their purities are at least $97 \%$ or higher. 1-Butanol was purchased from Junsei Chemicals, Japan. Toluene (99.5\%) and sodium metal were purchased from Samchun Chemicals, Korea. Hydrofluoric acid (Extrapure, DC Chemical Co.) and absolute ethanol (J. T. Baker Co.) were used as received. Toluene was further purified by distillation in sodium metal under $\mathrm{N}_{2}$. Ultrapure water $(18 \mathrm{MW}$ resistivity) was supplied by using a Barnstead ultra-pure water system. Glass surface was treated by using a plasma cleaner (PDC-32G, Harrick Plasma). Water contact angles were measured by using a SEO contact angle analyzer.

Glass substrate was soaked in an ultra- sonicator (20 minutes) including acetone for cleaning, and then blown dried with $\mathrm{N}_{2}$. The cleaned glass samples were treated with HF solution (HF $10 \mathrm{~mL}$ + Ultra-purewater $100 \mathrm{~mL}$ ) at six different conditions (no treatment, 1 second, 3 seconds, 5 seconds, 10 seconds, and 20 seconds). And then the samples were washed with ultra-pure water and blown dried with $\mathrm{N}_{2}$. As a final step of the surface treatment, HF-treated glass surface was further treated with Ar plasma under water vapor stream (32 W RF power; 500 mtorr vacuum pressure) for 5 minutes. The fully treated glass sample was dipped into APS solution (APS $0.12 \mathrm{~mL}+$ dry Toluene $60 \mathrm{~mL}$ ) for 1 hour to coat APS self-assembled monolayer (SAM) on the sample surface. Then, the sample was blown dried with $\mathrm{N}_{2}$ and further dried by heat for 5 mins. All of the SAM preparation was carried out in a glove box (Relative Humidity $<19 \%$ ). The APS-SAM surface was spin-coated with a mixed oxidant solution ( $3 \mathrm{wt} \% \mathrm{FeCl}_{3} 1.5 \mathrm{~g}$, and DUDO $0.2 \mathrm{~g}$ as a base inhibitor in 1-butanol $30 \mathrm{~mL}$ ) at $1,500 \mathrm{rpm}$ for 30 seconds. The mixed oxidant film was enclosed in an evaporation chamber and then EDOT molecules were vaporized at $70^{\circ} \mathrm{C}$ for 30 minutes, and hit the film surface to polymerize them on the surface. After that, the polymer thin film was annealed at $60^{\circ} \mathrm{C}$ under vacuum for 1 hour. Fig. 1 shows a schematic diagram of overall experimental procedure. In order to investigate the characteristics of PEDOT thin films, an optical microscope (Olympus, BX-51), Field EmissionScanning Electron Microscope (FEI, Sirion 200), and 4point probe (MStech, 4000) were used.

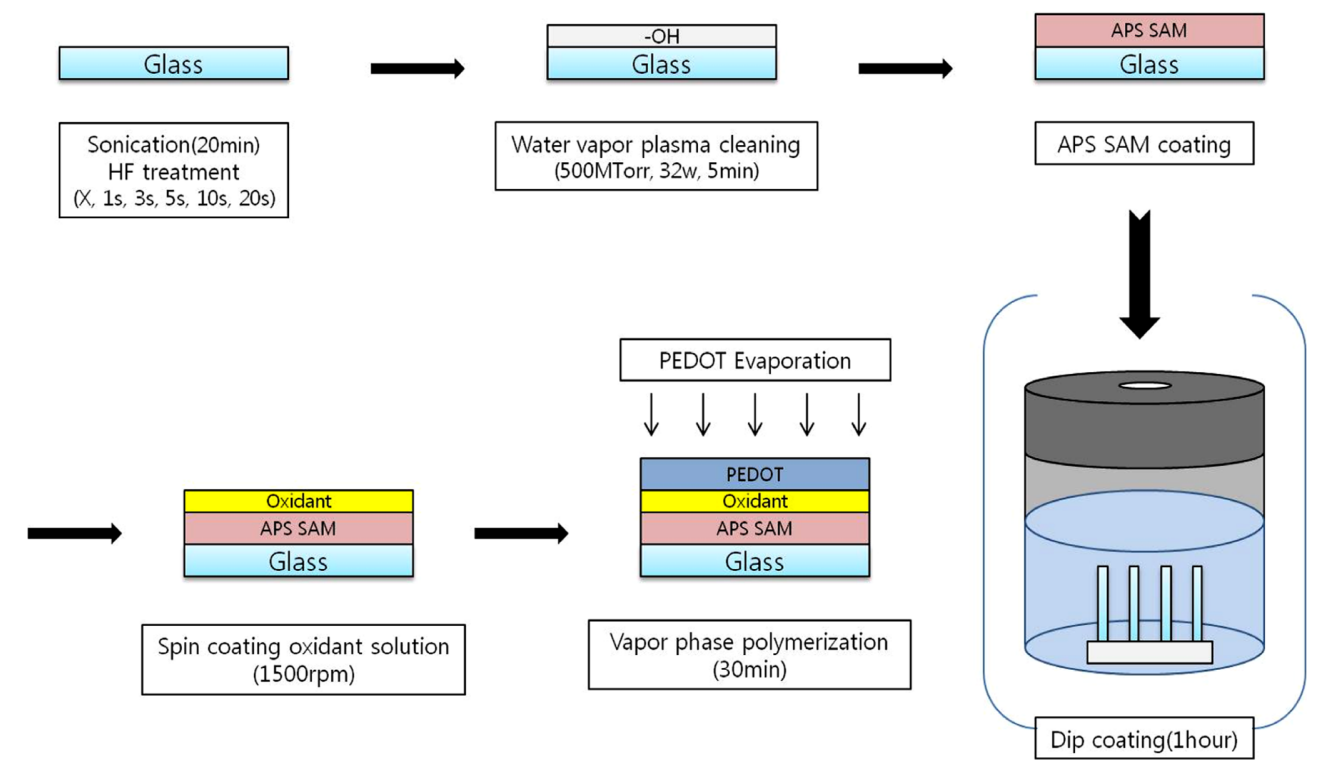

Fig. 1. Schematic diagram of experimental procedure 


\section{Results and Discussion}

Fig. 2 shows several optical microscope images of $\mathrm{FeCl}_{3}(3 \mathrm{wt} \% ; 1.5 \mathrm{~g})+$ polyurethane diol (DUDO $0.2 \mathrm{~g}$ ) oxidant films spin-coated (spin rate $=1500 \mathrm{rpm}$ for 30 seconds) on bare glass surfaces consecutively treated with $\mathrm{HF}$ and plasma. Images in Fig. 2 represent oxidant films on glass surfaces untreated (A), and also treated with HF for 1 second (B), 3 seconds (C), 5 seconds (D), 10 seconds (E), and 20 seconds (F), respectively, before plasma treatment. These images show a noticeable difference from those of oxidant films spin-coated on APS-SAM surfaces as shown in Fig. 2 of Part I study. All of the oxidant coated films in Fig. 2 were only partially formed except HF-untreated case. That is, the oxidant solution didn't wet the whole HF-treated glass surface. Accordingly, it is reasonable to expect that it will give rise to a poor quality PEDOT film after VPP. In fact, VPP-PEDOT films were only sparsely grown on the glass surfaces treated with HF prior to the plasma treatment as shown in Fig. 3. Images in Fig. 3 are PEDOT films directly grown on glass surfaces untreated, (A), and also treated with HF for 1 second

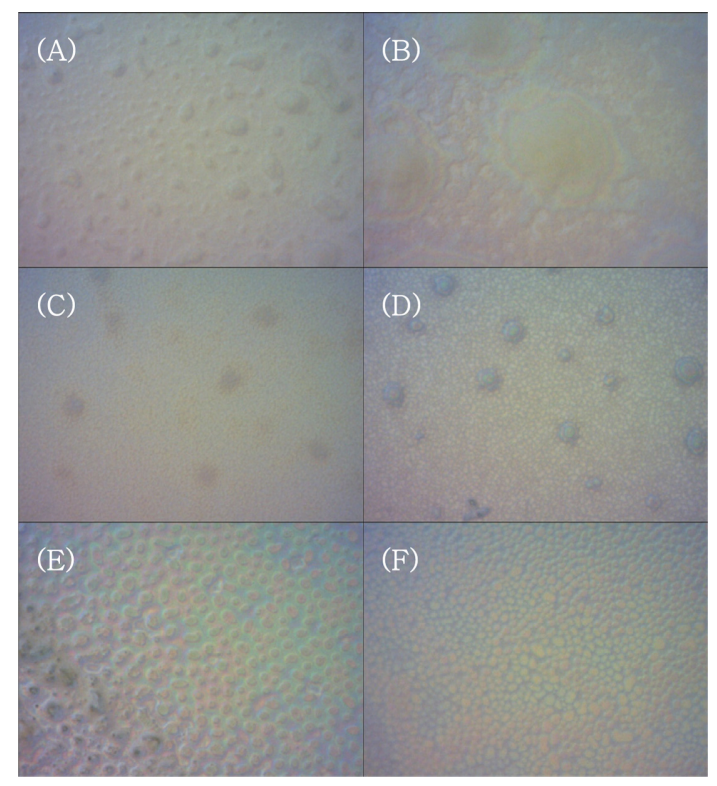

Fig. 2. Microscope images of oxidant films spin-coated from n-butanol $(30 \mathrm{~mL})$ solution including $\mathrm{FeCl}_{3}(1.5 \mathrm{~g})$ and $\operatorname{DUDO}(0.2 \mathrm{~g})$ on bare glass surfaces untreated (A), and treated with $\mathrm{HF}$ for $1 \mathrm{~s}(\mathrm{~B})$, for $3 \mathrm{~s}(\mathrm{C})$, for $5 \mathrm{~s}(\mathrm{D})$, for $10 \mathrm{~s}(\mathrm{E})$, and for 20s $(\mathrm{F})$ (Magnification $=500 \mathrm{x}$ )
(B), 3 seconds (C), 5 seconds (D), 10 seconds (E), and 20 seconds $(\mathrm{F})$, respectively, before plasma treatment and then spin coating of $\mathrm{FeCl}_{3}$ and DUDO mixed solution. Only dark areas in those images are PEDOT. It obviously proves that oxidant films were not uniformly coated on bare glass surfaces treated with HF and water vapor/Ar plasma successively. In addition, PEDOT film directly grown on the only plasma-treated and then oxidant-coated glass surface does not even fully cover the whole sample area (refer to Fig. 3(A)) even though $\mathrm{FeCl}_{3}$ oxidant mixed with DUDO was relatively evenly coated on the glass surface as shown in Fig. 2(A). Thus, in fact, any PEDOT film grown on water vapor/Ar plasma-treated surface was highly resistant to electrical conduction regardless of $\mathrm{HF}$ treatment. On the other hand, PEDOT thin films were grown on APS monolayer-coated glass surfaces and the surfaces were fully covered with PEDOT even if all of the film surfaces are quite irregular as shown in Fig. 4. For example, FESEM images in Fig. 3(C) and (D) of part I study are a PEDOT thin film on APS-SAM surface only treated with $\mathrm{H}_{2} \mathrm{O} / \mathrm{Ar}$ plasma prior to the monolayer coating.

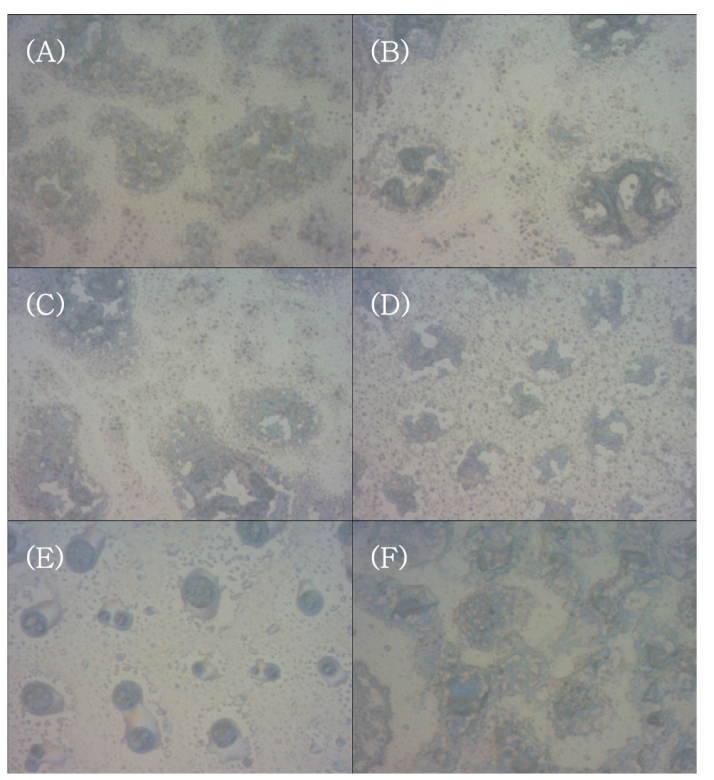

Fig. 3. Optical microscope images of PEDOT films on $\mathrm{FeCl}_{3}+$ DUDO oxidant mixture-coated glass surfaces. These images represent PEDOT films on bare glass surfaces untreated (A), and treated with $\mathrm{HF}$ for $1 \mathrm{~s}(\mathrm{~B}), 3 \mathrm{~s}(\mathrm{C})$, 5s (D), 10s (E), and 20s (F), respectively, prior to the plasma treatment. 


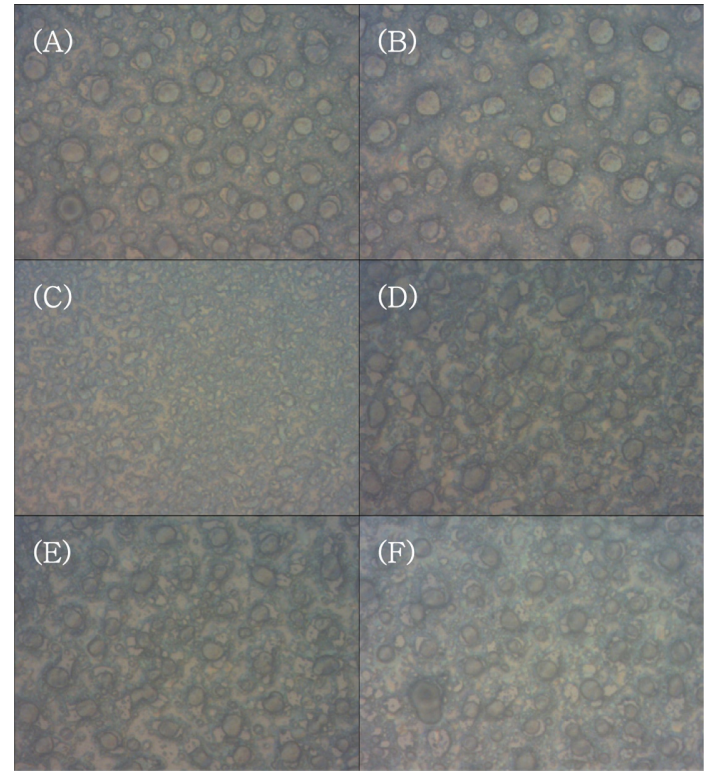

Fig. 4. Optical microscope images of PEDOT films on $\mathrm{FeCl}_{3}+$ DUDO oxidant mixture-coated APS-SAM surfaces. These images represent PEDOT films on APS-SAM surfaces untreated (A), and treated with HF for 1s (B), 3s (C), $5 \mathrm{~s}(\mathrm{D}), 10 \mathrm{~s}(\mathrm{E})$, and 20s (F), respectively, prior to the plasma treatment and monolayer coating.

They clearly show a fully covered film even if the surface is not smooth. However, at present, circular shaped protrusions existing on all of the PEDOT films shown in Fig. 4 are unknown yet. Further investigation is necessary. Sheet resistance values of PEDOT thin films in both HF-untreated and treated cases are listed in Table 1. Resistance was measured at several regions of each PEDOT sample. As shown, sheet resistance values in both untreated case and the case treated with HF for 20 seconds are nearly same and they range around $1210 \mathrm{O} /$ sq, which is the lowest in this study. However, in the

Table 1. Sheet Resistance (ohm/sq) values including standard deviations of PEDOT thin films on APS-SAM surfaces at various conditions of HF treatment.

\begin{tabular}{cc}
\hline HF Treatment Time & Sheet Resistance $(\mathrm{ohm} / \mathrm{sq})$ \\
\hline No HF treatment & $1217.911 \pm 364.689$ \\
1 second treatment & $1778.358 \pm 214.642$ \\
3 seconds treatment & $1372.623 \pm 184.954$ \\
5 seconds treatment & $1293.728 \pm 126.426$ \\
10 seconds treatment & $1308.804 \pm 172.425$ \\
20 seconds treatment & $1209.282 \pm 431.265$ \\
\hline
\end{tabular}

case treated with HF for 20 seconds, it was found that the value increases with time when it was measured by counting method while it did not change with time at all in HF-untreated but only plasma-treated case. It obviously indicates that the quality of PEDOT film in HF-treated case is poorer than in HF-untreated case.

Another notable thing is a strong jump of the resistance value after HF treatment for 1 second from an untreated state as shown in Fig. 5. In addition, the graph shows that the resistance value goes back to the initial level without HF treatment over 20 seconds. This trend is an acceptable result, considering the reaction mechanism of vitreous $\mathrm{SiO}_{2}$ surface with HF-based solution proposed by Knotter. ${ }^{[4]}$ According to the proposed mechanism, at a very early stage, when HF reacts with four oxygen-bridged silicon, $\mathrm{F}-\mathrm{SiO}_{3} \equiv$ is produced with the concomitant removal of $-\mathrm{OH}$ or $-\mathrm{H}_{2} \mathrm{O}^{+}$groups. Thereby, it results in a considerably hydrophobic surface. This proposition well explains why the glass surfaces treated with HF during a short period of time, such as 1 and 3 seconds, are poorly coated with the highly acidic oxidant solution as shown in Fig. 2(b) and (C). After the initial step of the mechanism, fluorinated silicon atoms become more fluorinated, generating and then finally removing $\operatorname{SiF}_{x}(x=1,2$, or 3$)$ units from the $\mathrm{SiO}_{2}$ matrix. Immediately following the removal of $\mathrm{SiF}_{\mathrm{x}}$ unit, silanol groups remain on the surface. This mechanism also well explains why the oxidant film is coated better in the case of 20 seconds treatment time (or even in the case of 10 seconds) than 1 second treatment time

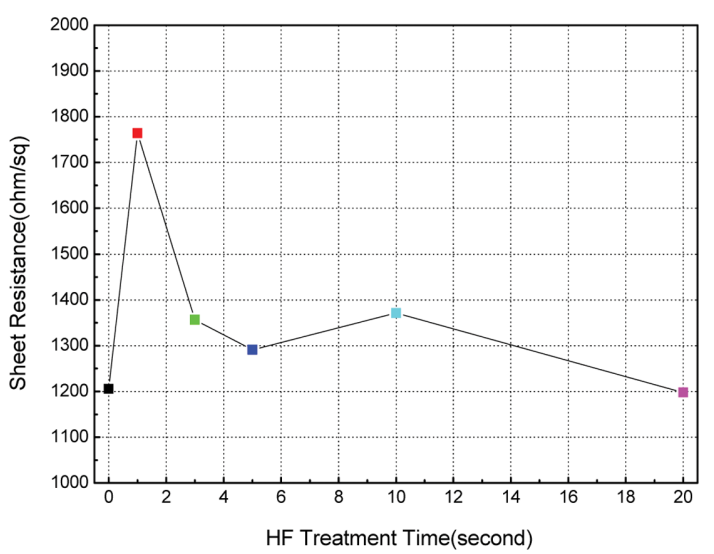

Fig. 5. Sheet Resistance (ohm/sq) values of PEDOT thin films on APS-SAM surfaces as a function of HF treatment time 
(refer to Fig. 2). However, this hypothesis can not satisfactorily explain the complicated relationship among a HF-treated degree of glass surface, plasma conditions, and the qualities of APS monolayer as well as PEDOT thin film. More detail investigation is underway.

\section{Conclusions}

This part II study clearly demonstrated that a well prepared APS-SAM on a glass surface treated with water vapor/Ar plasma is very useful for uniform coating of $\mathrm{FeCl}_{3}$ and DUDO mixed oxidant solution, regardless of $\mathrm{HF}$ treatment. On the other hand, a bare glass surface without APS-SAM but treated with HF and water vapor/Ar plasma generally led to a very poor oxidant film. As a result, PEDOT films vapor phasepolymerized on APS-SAM surfaces are much better, in the quality aspect, than those on bare glass surfaces. In particular, quality and electrical stability of PEDOT thin film, grown on APS-SAM surface treated only with plasma prior to the monolayer coating, is far superior to the film in the case treated with HF for 20 seconds even if both films have a similar level of sheet resistance.

\section{Acknowledgment}

This study was financially supported by Flexolution Corporation, Yongin, Korea.

\section{References}

[1] K. Kaneto, M. Maxfield, D. P.Nairns, A. G. MacDiarmid, and A. J. Heeger, "Electrochemistry of polyacetylene, $(\mathrm{CH}) \mathrm{x}$, Characteristics of polyacetylene cathodes", J. Chem. Soc., Faraday Trans. 1, Vol. 78, pp. 3417-3429, 1982.

[2] G. Gustafsson, Y. Cao, G. M. Tracy, F. Klavetter, N. Colaneri, and A. J. Heeger, "Flexible light-emitting diodes made from soluble conducting polymers", Nature, Vol. 357, pp. 477-479, 1993.

[3] S. Choi, W. Kim, and S. Kim, "Development of highly conductive poly(3,4-ethylenedioxythiophene) thin film using high quality 3-aminopropyltriethoxysilane self-assembled monolayer", J. Chosun Natural Sci., Vol. 4, pp. 294-297, 2011.

[4] D. M. Knotter, "Etching mechanism of vitreous silicon dioxide in HF-based solutions", J. Am. Chem. Soc., Vol. 122, pp. 4345-4351, 2000. 